Document downloaded from:

http://hdl.handle.net/10251/65718

This paper must be cited as:

Guirao Sánchez, AJ.; Montesinos Santalucia, V. (2015). Completeness in the Mackey topology. Functional Analysis and Its Applications. 49(2):97-105. doi:10.1007/s10688-0150091-2.

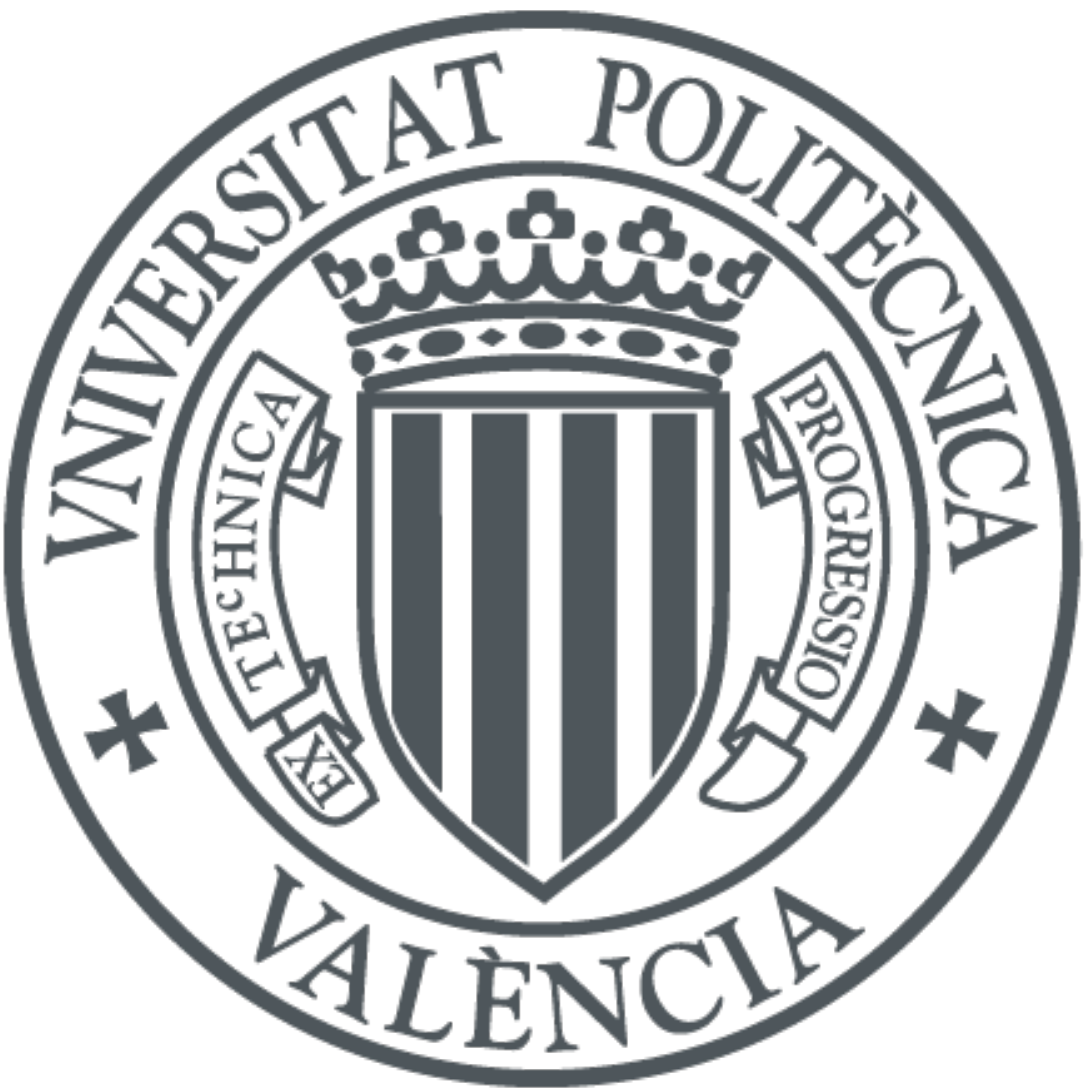

The final publication is available at

http://dx.doi.org/10.1007/s10688-015-0091-2

Copyright Springer Verlag

Additional Information 


\title{
Completeness in the Mackey topology
}

\author{
A. J. Guirao* and V. Montesinos ${ }^{\dagger}$
}

Dedicated to Václav Zizler on his $70^{\text {th }}$ birthday

\begin{abstract}
J. Bonet and B. Cascales $[\mathrm{BC}]$, answering a question of M. Kunze and W. Arendt, gave an example of a norming and norm-closed subspace $N$ of the dual of a Banach space $X$ such that $\mu(X, N)$ was not complete, where $\mu(X, N)$ denotes the Mackey topology associated to the dual pair $\langle X, N\rangle$. We prove in this note that we can decide on the completeness -in the positive or in the negative - of topologies of this form in a quite general context, thus providing large classes of examples to the aforesaid question.
\end{abstract}

\section{Introduction}

The Mackey topology $\mu(E, F)$ on $E$ associated to a dual pair $\langle E, F\rangle$ is the topology on $E$ of the uniform convergence on the family of all absolutely convex and $w(F, E)$-compact subsets of $F$, where $w(F, E)$ denotes the restriction to $F$ of the pointwise topology on $\mathbb{R}^{E}$ (i.e., the weak topology on $F$ associated to the dual pair $\langle E, F\rangle)$. The topology $\mu(E, F)$ is, by the Mackey-Arens theorem (see, e.g., [Ko, $\S 21.4(2)]$ or [FHHMZ, Thm. 3.41]), the strongest locally convex topology on $E$ that is compatible with the dual pair $\langle E, F\rangle$ (i.e., having the property that the dual space of $(E, \mu(E, F))$ is $F$ ). Of course, the norm topology of a normed space $X$ coincides with the Mackey topology $\mu\left(X, X^{*}\right)$, due to the $w^{*}$-compactness of the closed dual unit ball.

A topological vector space $(E, \mathcal{T})$ has an associated uniformity that induces its topology $\mathcal{T}$. This uniformity is uniquely determined if we require it to have a base of translation-invariant vicinities. This base is just $\left\{N_{U}: U \in \mathcal{B}\right\}$, where $\mathcal{B}$ is a base of absorbent circled neighborhoods of 0 in $(E, \mathcal{T})$, and $N_{U}:=\{(x, y) \in E \times E: y-x \in U\}$ for all $U \in \mathcal{B}$. Accordingly, every topological vector space can be embedded in a smallest complete topological vector space $(\widetilde{E}, \widetilde{\mathcal{T}})$, its completion, which is unique up to topological isomorphism (for this and related results, and for the terminology, see, e.g., [Ko, $\S 15.2$ and $\S 15.3])$. Grothendieck gave a characterization of the completion of a locally convex space (see, e.g., [Ko, §21.9]) that, when applied to $(E, \mu(E, F))$, reads:

(G) The completion of $(E, \mu(E, F))$ can be identified to the set of all linear functionals $f: F \rightarrow \mathbb{R}$ whose restriction $\left.f\right|_{K}$ to any absolutely convex and $w(F, E)$-compact subset $K$ of $F$ is $w(F, E)$ continuous.

In particular, $(E, \mu(E, F))$ is complete if, and only if, given a linear functional $f: F \rightarrow \mathbb{R}$ whose restriction to any absolutely convex and $w(F, E)$-compact subset of $F$ is $w(F, E)$-continuous, there is $x \in E$ such that $\left\langle x, x^{*}\right\rangle=f\left(x^{*}\right)$ for all $x^{*} \in F$. Observe that, if $X$ is a Banach space, then $\left(X^{*}, \mu\left(X^{*}, X\right)\right)$ is always complete, due to the fact that the norm-continuity of a linear functional

\footnotetext{
*Universidad Politécnica de Valencia. Instituto de Matemática Pura y Aplicada. Camino de Vera, s/n, 46022 Valencia, Spain. Supported in part by MICINN and FEDER (Project MTM2008-05396), by Fundación Séneca (Project 08848/PI/08), by Generalitat Valenciana (GV/2010/036), and by Universidad Politécnica de Valencia (Project PAID-06-09-2829). email: anguisa2@mat.upv.es

${ }^{\dagger}$ Universidad Politécnica de Valencia. Instituto de Matemática Pura y Aplicada. Camino de Vera, s/n, 46022 Valencia, Spain. Supported in part by Project MICINN MTM2011-22417, Generalitat Valenciana (GV/2010/036), and by Universidad Politécnica de Valencia (Project PAID-06-09-2829). email: vmontesinos@ mat.upv.es.

AMS classification: Primary: 46B10. Secondary: 54H05. Keyword and phrases: Mackey-star topology, completeness, local completeness, Banach space.
} 
$f: X \rightarrow \mathbb{R}$ can be checked on absolutely convex and $w\left(X, X^{*}\right)$-compact subsets of $X$ (a consequence of Krein's theorem). For these results see, e.g., [Ko] or [FHHMZ].

J. Bonet and B. Cascales [BC], answering a question by M. Kunze and W. Arendt, gave an example of a norm-closed and 1-norming subspace $P$ of the dual $X^{*}$ of a Banach space $X$ such that $(X, \mu(X, P))$ was not complete (a norming subspace of the dual of a Banach space $X$ is a subspace $P$ of $X^{*}$ such that there exists $C>0$ such that $\|x\| \leq C \sup \left\{\left\langle x, x^{*}\right\rangle: x^{*} \in P,\|x\| \leq 1\right\}$ for all $x \in X$; it is called 1-norming if $C=1$ ). The example was $P:=C[0,1]$, the space of all continuous functions on $[0,1]$, seen as a closed subspace of the dual space $X^{*}:=\ell_{\infty}[0,1]$ of the space $X:=\left(\ell_{1}[0,1],\|\cdot\|_{1}\right)$. They used Krein's theorem together with the description of the closed unit ball of $C[0,1]^{*}$.

It was natural to search for a quite general rule providing "well located" norm-closed and $w^{*}$-dense subspaces $Y$ of the dual of a Banach for which $\mu(X, Y)$ is -or is not- complete. By "well located" we mean in between a predual —if any — and the dual space of $X$ (after all, $\ell_{1}[0,1]$ has a predual $c_{0}[0,1]$, and the space $C[0,1]$ used in $[\mathrm{BC}]$ has no relation whatsoever to $\left.c_{0}[0,1]\right)$. Our approach gives a recipe for $\|\cdot\|_{\infty}$-closed subspaces $Y$ of $\ell_{\infty}(\Gamma)$ that contain $c_{0}(\Gamma)$ and such that $\mu\left(\ell_{1}(\Gamma), Y\right)$ is - or is not- complete (see Corollary 6). It works, too, for general Banach spaces $X$ and the topology $\mu(X, Y)$ where $Y$ is a norm-closed and $w^{*}$-dense subspace of $X^{*}$-for example, a predual $Y$ if available - (Theorem 1 and, in the separable case, Corollary 3). Since $c_{0}$ is the simplest example of a Banach space having no predual, the natural question after our discussion was whether the most accessible $\|\cdot\|_{1}$-closed and $w^{*}$-dense subspaces $H$ of the dual $\ell_{1}$-namely, the hyperplanes given as the kernel of an element in $\ell_{\infty} \backslash c_{0}$ - give a complete or uncomplete $\mu\left(c_{0}, H\right)$ topology. The answer is in Corollary 14. We finalize this note by considering the special case of a Banach space that has codimension 1 in its bidual —as in the case of James' space $J$.

Our notation is standard. In the locally convex space setting we adopt the Banach space terminology (for example, if $E$ is a locally convex space then $E^{*}$ denotes its topological dual, and if $\langle E, F\rangle$ is a dual pair then $w(E, F)$ denotes the topology on $E$ of the pointwise convergence on elements in $F$ ). Given a subset $S$ of a vector space $E$, we put $\Gamma(S)$ for the absolutely convex hull of $S$. i.e., $\Gamma(S):=\left\{x \in X: x=\sum_{i=1}^{n} \lambda_{i} x_{i}, x_{i} \in S\right.$ for $i=1,2, \ldots, n, n \in \mathbb{N}$, and $\left.\sum_{i=1}^{n}\left|\lambda_{i}\right| \leq 1\right\}$. A subset $A$ of a vector space is said to be absolutely convex if $\Gamma(A)=A$. If $X$ is a Banach space and $Y$ is a subspace of $X$, then $B_{Y}$ denotes the unit ball of $Y$, i.e., $B_{Y}:=Y \cap B_{X}$, where $B_{X}$ is the closed unit ball of $X$. For other non-defined concepts or non-stated results we refer, e.g., to [FHHMZ].

\section{Completeness for the Mackey topology with respect to some subspaces of the dual space}

Let $X$ be a Banach space. The topology $\mu\left(X, X^{*}\right)$ coincides with the topology induced by the norm, and so it is complete. Assume that $X$ has a predual $P \subset X^{*}$ (a particular case of a 1-norming -and so $w\left(X^{*}, X\right)$-dense- subspace of $\left.X^{*}\right)$. We mentioned in the Introduction that $\mu(X, P)$ is then complete. The question raised by M. Kunze and W. Arendt was whether $\mu(X, Y)$ was complete for every norm-closed and $w\left(X^{*}, X\right)$-dense subspace $Y$ of $X^{*}$. Observe that even in the case of the existence of a predual $P$, the $\mu(X, Y)$-completeness of $X$ for a norm-closed subspace $Y$ such that $P \subset Y \subset X^{*}$ is not guaranteed. It is true that if $(E, \mathcal{T})$ is a complete locally convex space and $\mathcal{T}^{\prime}$ is a stronger locally convex topology on $E$ with a base of $\mathcal{T}$-closed neighborhoods of 0 , then $\left(E, \mathcal{T}^{\prime}\right)$ is also complete (see, e.g., [Ko, $\left.\S 18.4(4)\right]$ ); however, there is in general no reason for $Y$ as above to ensure that $\mu(X, Y)$ has a base of $\mu(X, P)$-closed neighborhoods. A strengthening of the Kunze-Arendt question is then whether $\mu(X, Y)$ was complete for every norm-closed subspace of the dual in between a predual $P$-if any- and $X^{*}$. The example $Y$ in [BC] was not located this way. 


\subsection{The main result and some consequences}

Let $X$ be a Banach space. Let $P$ be a $w^{*}$-dense and norm-closed subspace of $X^{*}$. Let $\mathcal{K}:=$ $\left\{K: K\right.$ an absolutely convex and $w^{*}$-compact subset of $\left.X^{*}\right\}$. Put

$$
S:=\left\{x^{*} \in X^{*}: \text { there exists } K \in \mathcal{K} \text { such that } K \subset P \oplus \operatorname{span}\left\{x^{*}\right\}, \text { and } x^{*} \in \overline{K \cap P} w^{*}\right\} .
$$

The set $S$ certainly contains $P$.

Regarding (i) in the next result, we refer to what was said at the beginning of this section. Concerning (ii), note that the sum of a closed subspace and a finite-dimensional subspace of a normed space is always closed. In general, the sum of two closed subspaces of a normed space is not closed.

Theorem 1 Let $X$ be a Banach space, and let $P$ be a norm-closed and $w^{*}$-dense subspace of $X^{*}$. The following two statements hold:

(i) Assume that $(X, \mu(X, P))$ is complete. Then, if $S_{0} \subset S$ and $Y:=\overline{P \oplus \operatorname{span}\left(S_{0}\right)}\|\cdot\|$, the space $(X, \mu(X, Y))$ is complete, too.

(ii) If $x^{*} \in X^{*} \backslash S$, then $\left(X, \mu\left(X, P \oplus \operatorname{span}\left\{x^{*}\right\}\right)\right)$ is not complete.

We shall prove later (see (ii) in Corollary 6 and Remark 7) that (ii) in Theorem 1 is not an empty statement: There are Banach spaces $X$ such that the set $S$ defined in (1) above is a proper subset of the space $X^{*}$.

The nature of the set $S$ defined in (1) above is somehow elusive. The following result shows that, for norm-closed and $w^{*}$-dense subspaces $P$ of $X^{*}$, the set $S$ contains a natural subspace of $X^{*}$, namely the subspace consisting of all the $w^{*}$-limits of $w^{*}$-Cauchy sequences in $P$. As a consequence we obtain, in the separable case, a characterization of $S$ (proof of Corollary 3). For another instance where $S$ can be easily identified see Corollary 6 and the subsequent Remark 7.

Proposition 2 Let $X$ be a Banach space, and let $P$ be a $w^{*}$-dense and norm-closed subspace of $X^{*}$.Put

$$
Z:=\left\{x^{*} \in X^{*}: \text { there exists a sequence }\left\{p_{n}^{*}\right\}_{n=1}^{\infty} \text { in P such that } p_{n}^{*} \stackrel{w^{*}}{\rightarrow} x^{*}\right\} .
$$

Then

(i) $Z \subset S$, where $S$ is the subset of $X^{*}$ defined in (1).

(ii) If $X$ is separable, we have $Z=S$.

For separable Banach spaces, Theorem 1 and Proposition 2 lead to the following result. In fact, the proof below shows that the set $S$ defined in (1) coincides with $X^{*}$, and the result then follows from (i) in Theorem 1.

Corollary 3 Let $X$ be a separable Banach space. Let $P$ be a norm-closed and norming subspace of $X^{*}$ such that $(X, \mu(X, P))$ is complete. Then $(X, \mu(X, Y))$ is complete for every norm-closed subspace $Y$ of $X^{*}$ such that $P \subset Y$.

Remark 4 Corollary 3 applies, for example, to separable Banach spaces $X$ having a predual $P$. The separability requirement can be removed as soon as $P$ is $w^{*}$-sequentially dense in $Y$.

\subsection{Proofs}

\section{Proof of Theorem 1.}

(i) We Claim that given a linear map $f: Y \rightarrow \mathbb{R}$ such that $\left.f\right|_{K}$ is $w^{*}$-continuous for every $K \in \mathcal{K}$ with $K \subset Y$, there exists $x \in X$ such that $\left\langle x, y^{*}\right\rangle=f\left(y^{*}\right)$ for all $y^{*} \in Y$. Once this Claim is proved, an application of Grothendieck's completeness criterion $(\mathrm{G})$ above will finalize the proof of statement (i).

Step 1: Observe first that such an $f$ is norm-continuous. Indeed, let $\left\{y_{n}^{*}\right\}_{n=1}^{\infty}$ be a norm-null sequence in $Y$. Thus, $A:=\left\{y_{n}^{*}: n \in \mathbb{N}\right\} \cup\{0\}$ is a norm-compact subset of $X^{*}$. The completeness 
of $\left(X^{*},\|\cdot\|\right)$ and Krein's theorem show that $B:=\overline{\Gamma(A)}\|\cdot\|(\subset Y)$ is norm-compact. Since $w^{*}$ and the norm topology agree on $B$, and $\left.f\right|_{K}$ is $w^{*}$-continuous, we get that $f\left(y_{n}^{*}\right) \rightarrow 0$, and $f$ is thus norm-continuous, as claimed.

Step 2: We shall prove the Cla im for the norm-closed subspace $Y:=P \oplus \operatorname{span}\left\{x^{*}\right\}$, where $x^{*} \in S$. Observe that, in particular, $\left.f\right|_{K}$ is $w^{*}$-continuous for every $K \in \mathcal{K}, K \subset P$. The completeness of $\mu(X, P)$ implies the existence of $x \in X$ such that $\left\langle x, p^{*}\right\rangle=f\left(p^{*}\right)$ for all $p^{*} \in P$. Since $x^{*} \in S$, we can find $K \in \mathcal{K}, K \subset P \oplus \operatorname{span}\left\{x^{*}\right\}$, such that $x^{*} \in \overline{K \cap P} w^{*}$. Let $\left\{p_{i}^{*}: i \in I, \leq\right\}$ be a net in $K \cap P$ such that $p_{i}^{*} \stackrel{w^{*}}{\rightarrow} x^{*}$. Since $\left\{p_{i}^{*}: i \in I\right\} \cup\left\{x^{*}\right\}$ is a subset of $K$ we get $f\left(p_{i}^{*}\right) \rightarrow f\left(x^{*}\right)$. Simultaneously, $\left(f\left(p_{i}^{*}\right)=\right)\left\langle x, p_{i}^{*}\right\rangle \rightarrow\left\langle x, x^{*}\right\rangle$, so we conclude $\left\langle x, x^{*}\right\rangle=f\left(x^{*}\right)$, and so $\left\langle x, y^{*}\right\rangle=$ $f\left(y^{*}\right)$ for all $y^{*} \in Y$. This proves the Claim in this case.

Step 3: Let $Y:=P \oplus \operatorname{span}\left\{S_{0}\right\}$, where $S_{0} \subset S$. The validity of the Claim in this case follows from Step 2. Indeed if $y^{*} \in Y$, we can find a finite subset $S_{00}$ such that $y^{*} \in P \oplus \operatorname{span}\left\{S_{00}\right\}$. Step 2 shows the existence, for each $y_{0}^{*} \in S_{00}$, of an element $x\left(y_{0}^{*}\right) \in X$ such that $\left\langle x\left(y_{0}^{*}\right), z^{*}\right\rangle=f\left(z^{*}\right)$ for all $z^{*} \in P \oplus\left\{y_{0}^{*}\right\}$. Since $P$ is $w^{*}$-dense, the exists $x \in X$ such that $x=x\left(y_{0}^{*}\right)$ for all $y_{0}^{*} \in S_{00}$, and thus $\left\langle x, y^{*}\right\rangle=f\left(y^{*}\right)$ for all $y^{*} \in Y$.

Step 4: Finally, take $z^{*} \in Y:=\overline{P \oplus \operatorname{span}\left\{S_{0}\right\}}{ }^{\|\cdot\|}$, where $S_{0} \subset S$. Then, there exists a sequence $\left\{z_{n}^{*}\right\}$ in $P \oplus \operatorname{span}\left(S_{0}\right)$ converging in norm to $z^{*}$. By Step 3, there exists $x \in X$ such that $f\left(z_{n}^{*}\right)=$ $\left\langle x, z_{n}^{*}\right\rangle$ for every $n \in \mathbb{N}$. The norm-continuity of $f$ (see Step 1) allows us to take limits and obtain that $f\left(z^{*}\right)=\left\langle x, z^{*}\right\rangle$, which concludes the proof.

(ii) Observe that the algebraic direct sum $Y:=P \oplus \operatorname{span}\left\{x^{*}\right\}$ is a norm-topological direct sum in $X^{*}$. In order to prove (ii) we shall define a $w^{*}$-discontinuous linear mapping $f: Y \rightarrow \mathbb{R}$ such that $\left.f\right|_{K}$ is $w^{*}$-continuous for every $K \in \mathcal{K}, K \subset Y$. This will show (ii) by Grothendieck's completeness criterion (G) above. It is enough to take $f: Y \rightarrow \mathbb{R}$ such that $\left.f\right|_{P} \equiv 0$ and $f\left(x^{*}\right) \neq 0$. Since $x^{*} \in \bar{P}^{w^{*}}$, certainly $f$ is $w^{*}$-discontinuous on $Y$. Fix $K \in \mathcal{K}$ such that $K \subset Y$. A lemma due to Grothendieck (see, e.g., [Ko, $\S 21.6(5)]$ ) ensures that to prove the $w^{*}$-continuity of $\left.f\right|_{K}$ it is enough to check the $w^{*}$-continuity at 0 of $\left.f\right|_{K}$. Let $\left\{x_{\delta}^{*}: \delta \in \Delta, \leq\right\}$ be then a $w^{*}$-null net in $K$, and let $\left\{x_{i}^{*}: i \in I, \leq\right\}$ be an arbitrary subnet of $\left\{x_{\delta}^{*}: \delta \in \Delta, \leq\right\}$. Put $x_{i}^{*}=p_{i}^{*}+\lambda_{i} x^{*}$, where $p_{i}^{*} \in P$ and $\lambda_{i} \in \mathbb{R}$ for all $i \in I$. Since $K$ is a bounded set, so it is the set $\left\{p_{i}^{*}: i \in I\right\}\left(\subset X^{*}\right)$ and the set $\left\{\lambda_{i}: i \in I\right\}$ (say $\left|\lambda_{i}\right| \leq M$ for all $i \in I$ and some $M>0$ ). There exists then a subnet $\left\{p_{i_{j}}^{*}: j \in J, \leq\right\}$ of $\left\{p_{i}: i \in I, \leq\right\}$ that $w^{*}$-converges to an element $z^{*} \in X^{*}$. This shows that $\left\{\lambda_{i_{j}}: j \in J, \leq\right\}$ converges to some $\lambda_{0} \in \mathbb{R}$ and so $0=z^{*}+\lambda_{0} x^{*}$. We claim that $\lambda_{0}=0$. Assume not. The set $B:=K / \lambda_{0}+\left[-M /\left|\lambda_{0}\right|, M /\left|\lambda_{0}\right|\right] x^{*}$ is an absolutely convex and $w^{*}$-compact subset of $Y$. Note that

$$
\frac{-1}{\lambda_{0}} p_{i_{j}}^{*}=\frac{-1}{\lambda_{0}} x_{i_{j}}^{*}+\frac{\lambda_{i_{j}}}{\lambda_{0}} x^{*} \rightarrow x^{*} \text { in the } w^{*} \text { topology, }
$$

and that

$$
\frac{-1}{\lambda_{0}} x_{i_{j}}^{*}+\frac{\lambda_{i_{j}}}{\lambda_{0}} x^{*} \in B \cap P \text { for all } j \in J .
$$

This contradicts the fact that $x^{*} \notin S$. The claim is proved, so $\lambda_{0}=0$. Then $f\left(x_{i_{j}}^{*}\right)=f\left(p_{i_{j}}^{*}\right)+$ $\lambda_{i_{j}} f\left(x^{*}\right)=\lambda_{i_{j}} f\left(x^{*}\right) \rightarrow 0$. Since the subnet $\left\{x_{i}^{*}: i \in I, \leq\right\}$ of $\left\{x_{\delta}^{*}: \delta \in \Delta, \leq\right\}$ was arbitrary, we get $f\left(x_{\delta}^{*}\right) \rightarrow 0$, as we wanted to show. This proves, finally, that $(X, \mu(X, Y))$ is not complete.

Proof of Proposition 2. (i) Given $x^{*} \in Z$ find a sequence $\left\{p_{n}^{*}\right\}_{n=1}^{\infty}$ in $P$ such that $p_{n}^{*} \stackrel{w^{*}}{\rightarrow} x^{*}$. Put $Y:=P \oplus \operatorname{span}\left\{x^{*}\right\}$. As it was mentioned above, $Y$ is a norm-closed subspace of $X^{*}$. The set $K:=\overline{\Gamma\left(\left\{p_{n}^{*}: n \in \mathbb{N}\right\}\right)} w^{*}$, being a $w^{*}$-closed and bounded subset of $X^{*}$, is $w^{*}$-compact. We claim that $K \subset Y$. Since $x^{*} \in \overline{K \cap P} w^{*}$, this will show that $x^{*} \in S$. The following argument proves the claim; it uses an idea from [Ko, $§ 20.9$ (6)] (see also [Fl, p. 17, Lemma]). Put $d_{n}^{*}:=x^{*}-p_{n}^{*}$ for $n \in \mathbb{N}$. Since $\left\{d_{n}^{*}\right\}_{n=1}^{\infty}$ is a $w^{*}$-null (hence norm-bounded) sequence, we can define a bounded linear mapping $T: X \rightarrow c_{0}$ by $T x:=\left(\left\langle x, d_{n}^{*}\right\rangle\right)$ for $x \in X$. The adjoint mapping $T^{*}: \ell_{1} \rightarrow X^{*}$ is 
then bounded and $w^{*}-w^{*}$-continuous. Observe that $T^{*}\left(e_{n}^{*}\right)=d_{n}^{*}$ for all $n \in \mathbb{N}$, where $e_{n}^{*}$ denotes the $n$-th canonical basis vector in $\ell_{1}$. Note that

$$
\left\{d_{n}^{*}: n \in \mathbb{N}\right\} \subset T^{*}\left(B_{\ell_{1}}\right)=T^{*}\left(\overline{\Gamma\left(\left\{e_{n}^{*}: n \in \mathbb{N}\right\}\right)}\|\cdot\|\right) \subset \overline{\Gamma\left(\left\{d_{n}^{*}: n \in \mathbb{N}\right\}\right)}{ }^{\|\cdot\|} \subset Y .
$$

Since $T^{*}\left(B_{\ell_{1}}\right)$ is $w^{*}$-compact, we get then $\overline{\Gamma\left(\left\{d_{n}^{*}: n \in \mathbb{N}\right\}\right)} w^{*} \subset Y$. From this, it follows easily that $K \subset Y$.

(ii) Assume now that $X$ is separable. Let $x^{*} \in S$, and let $K$ be an absolutely convex and $w^{*}$-compact subset of $P \oplus \operatorname{span}\left\{x^{*}\right\}$ such that $x^{*} \in \overline{K \cap P} w^{*}$. Since $X$ is separable, $\left(K, w^{*}\right)$ is metrizable; thus, we can find a sequence $\left\{p_{n}^{*}\right\}_{n=1}^{\infty}$ in $K \cap P$ that $w^{*}$-converges to $x^{*}$. This proves that $x^{*} \in Z$.

Proof of Corollary 3. Due to the fact that $P$ is norming, there exists $\alpha>0$ and $\beta>0$ such that $B_{X^{*}} \subset \alpha \overline{B_{P}} w^{*} \subset \beta B_{X^{*}}$, where $B_{P}$ denotes the closed unit ball of $P$. Fix $x^{*} \in B_{X^{*}}$. Since $X$ is separable, $\left(B_{X^{*}}, w^{*}\right)$ is metrizable, hence there exists a sequence $\left\{p_{n}^{*}\right\}_{n=1}^{\infty}$ in $\alpha B_{P}$ such that $p_{n}^{*} \stackrel{w^{*}}{\rightarrow} x^{*}$. This shows that $Z=X^{*}$, where $Z$ was defined in (2). By Proposition $2, S=X^{*}$, and an appeal to (i) in Theorem 1 finishes the argument.

Remark 5 The argument behind the proof of Proposition 2 is conceptually simple. A shorter onealthough based on a much more delicate result - follows (we use the notation above): The set $K:=\overline{\Gamma\left(\left\{p_{n}^{*}: n \in \mathbb{N}\right\}\right)} w^{*}$ is $w^{*}$-compact. If $A:=\left\{p_{n}^{*}: n \in \mathbb{N}\right\} \cup\left\{x^{*}\right\}$, then clearly $B:=A \cup(-A)$ is a (countable) James boundary of $K$. By a result of Godefroy [G] (see also [FHHMZ, Theorem 3.122], we have that $B$ is in fact a strong boundary, i.e., $K=\overline{\operatorname{conv}(B)}^{\|\cdot\|}$, hence $K \subset Y$.

\subsection{Some particular examples}

In the next result, we consider the spaces $X:=\ell_{1}(\Gamma)$ and $P:=c_{0}(\Gamma)$ for an infinite - countable or uncountable- set $\Gamma$. Part (ii) gives examples of 1-norming norm-closed subspaces $Y$ of the dual of the Banach space $\ell_{1}(\Gamma)$ such that $\mu\left(\ell_{1}(\Gamma), Y\right)$ is not complete, and yet $Y$ contains $c_{0}(\Gamma)$.

Corollary 6 The following statements hold:

(i) Let $S_{0}$ be a subset of the set $S$ in $\ell_{\infty}(\Gamma)$, and let $Y:=\overline{c_{0}(\Gamma) \oplus \operatorname{span}\left\{S_{0}\right\}}\|\cdot\|$. Then the space $\left(\ell_{1}(\Gamma), \mu\left(\ell_{1}(\Gamma), Y\right)\right)$ is complete. In particular, the space $\left(\ell_{1}(\Gamma), \mu\left(c_{0}(\Gamma) \oplus \operatorname{span}\left\{x^{*}\right\}\right)\right)$ is complete for every $x^{*} \in \ell_{\infty}(\Gamma)$ such that $\operatorname{supp} x^{*}$ is countable.

(ii) Let $\Gamma$ be an uncountable set. Let $x^{*} \in \ell_{\infty}(\Gamma)$ such that $\operatorname{supp} x^{*}$ is uncountable. Then the space $\left(\ell_{1}(\Gamma), \mu\left(\ell_{1}(\Gamma), c_{0}(\Gamma) \oplus \operatorname{span}\left\{x^{*}\right\}\right)\right)$ is not complete.

Proof. The first part of (i) here follows straightforwardly from (i) in Theorem 1 . Indeed, $P:=$ $c_{0}(\Gamma)\left(\subset \ell_{\infty}(\Gamma)\right)$ has the property that $P^{*}=\ell_{1}(\Gamma)$, and then it is a $\|\cdot\|_{\infty}$-closed and $w^{*}$-dense subspace of $\ell_{\infty}(\Gamma)$ such that $\mu\left(\ell_{1}(\Gamma), c_{0}(\Gamma)\right)$ is complete. To prove the particular case, fix $x^{*} \in$ $\ell_{\infty}(\Gamma)$ such that supp $x^{*}$ is countable. We shall prove that $x^{*} \in S$, and the result will follow. Since $B_{c_{0}}$ is $w^{*}$-dense in $B_{\ell_{\infty}}$, and $\left(B_{\ell_{\infty}}, w^{*}\right)$ is metrizable, there exists a sequence $\left\{c_{n}^{*}\right\}_{n=1}^{\infty}$ in $\left\|x^{*}\right\| \cdot B_{c_{0}(\Gamma)}$ that $w^{*}$-converges to $x^{*}$. This shows that $x^{*} \in Z$, where $Z$ was defined in (2), hence, by (i) in Proposition $2, x^{*} \in S$.

(ii) Put $X:=\ell_{1}(\Gamma)$ and fix $x^{*} \in \ell_{\infty}(\Gamma)$ such that supp $x^{*}$ is uncountable. Put $Y:=c_{0}(\Gamma) \oplus\left\{x^{*}\right\}$. Assume that $x^{*} \in S$. Then we can find $K \in \mathcal{K}$ such that $K \subset Y$ and $x^{*} \in \overline{K \cap c_{0}(\Gamma)} w^{*}$. Observe that there exists $\varepsilon>0$ such that the set $\Gamma_{\varepsilon}:=\left\{\gamma \in \Gamma:\left|x^{*}(\gamma)\right|>\varepsilon\right\}$ is uncountable. Indeed, if not the set $\Gamma_{1 / n}$ should be countable for all $n \in \mathbb{N}$, and so supp $x^{*}$ will be also countable, a contradiction. Fix a countable infinite subset $N$ of $\Gamma_{\varepsilon}$. We can then find a sequence $\left\{c_{n}^{*}\right\}_{n=1}^{\infty}$ in $K \cap c_{0}(\Gamma)$ that converges to $x^{*}$ pointwise on $N$. The sequence $\left\{c_{n}^{*}\right\}_{n=1}^{\infty}$ is bounded in $\ell_{\infty}(\Gamma)$, so it has a $w^{*}$-cluster point $x_{N}^{*} \in \ell_{\infty}(\Gamma)$. Several facta about $x_{N}^{*}$ should be clear: (a) supp $x_{N}^{*}$ is countable. (b) $\left|x_{N}^{*}(\gamma)\right| \geq \varepsilon$ for all $\gamma \in N$ (in particular, $x_{N}^{*} \notin c_{0}(\Gamma)$ ). (c) $x_{N}^{*} \in K(\subset Y)$, hence 
$x_{N}^{*}=c^{*}+\lambda x^{*}$, where $c^{*} \in c_{0}(\Gamma)$ and $\lambda \neq 0$. Certainly, (a), (b), and (c) simultaneously are in contradiction. It follows then that $x^{*} \notin S$ and we can apply (ii) in Theorem 1.

Remark 7 The proof of Corollary 6 identifies the set $S$ defined in (1) in the case of the subspace $P:=c_{0}(\Gamma)$ of $\ell_{\infty}(\Gamma)$ for an uncountable set $\Gamma$ (for countable $\Gamma$ we refer to the paragraph before Corollary 3), a set $S$ that turns out to be the proper closed subspace of $\ell_{\infty}(\Gamma)$ consisting of all its countably supported vectors.

\section{Dualizing completeness and some examples}

This section is instrumental, and will be used in the analysis of completeness for Mackey topologies of the form $\mu\left(c_{0}, H\right)$, where $H$ is a $\|\cdot\|_{1}$-closed hyperplane of $\ell_{1}$, or $\mu(J, H)$, where $J$ is James' space and $H$ is a norm-closed and norming subspace of its dual space, see Section 4 . Results here relate completeness in the space to completeness in the dual. Lemma 9 is formulated for the space $c_{0}$, and carries completeness from the dual to the space. Lemma 10 is more general, and acts in the opposite direction.

For notions and results needed in this section we refer, e.g., to [BP]. Let $E$ be a locally convex space, and let $E^{*}$ be its topological dual. A disc is an absolutely convex and bounded subset $B$ of $E$. Every disc $B$ generates a normed space $\left(E_{B},\|\cdot\|_{B}\right)$, where $E_{B}:=\bigcup_{n=1}^{\infty} n B$ and $\|\cdot\|_{B}$ is the Minkowski functional of $B$ on $E_{B}$, i.e., $\|x\|_{B}:=\inf \{\lambda>0: x \in \lambda B\}$ for $x \in E_{B}$. A disc is called a Banach disc if $\left(E_{B},\|\cdot\|_{B}\right)$ is a Banach space. A sequence $\left\{x_{n}\right\}_{n=1}^{\infty}$ in $E$ is said to be locally Cauchy (locally convergent) if there exists a disc $B$ in $E$ such that $\left\{x_{n}\right\}_{n=1}^{\infty}$ is a $\|\cdot\|_{B}$-Cauchy sequence in $E_{B}$ (respectively, it is $\|\cdot\|_{B}$-convergent to an element $x \in E_{B}$-if $x:=0$ we say, in this case, that the sequence $\left\{x_{n}\right\}_{n=1}^{\infty}$ is locally null). The space $E$ is said to be locally complete if every locally Cauchy sequence in $E$ is locally convergent. The following standard results will be used below.

(A) The space $E$ is locally complete if, and only if, every closed disc in $E$ is a Banach disc [BP, Proposition 5.1.6].

(B) The quality of $E$ being locally complete depends only on the dual pair $\left\langle E, E^{*}\right\rangle[\mathrm{BP}$, Corollary 5.1.7].

(C) Every sequentially complete locally convex space is locally complete [BP, Corollary 5.1.8].

We also quote here another result ([BP, Theorem 5.1.11]) in the theory of locally convex spaces that shall be used in several arguments below.

Theorem 8 Let $(E, \mathcal{T})$ be a locally convex space. Then, the following conditions are equivalent:

(i) $(E, \mathcal{T})$ is locally complete.

(ii) The closed absolutely convex hull of every locally null sequence in $E$ is $\mathcal{T}$-compact.

(iii) The closed absolutely convex hull of every $w\left(E, E^{*}\right)$-null sequence is $w\left(E, E^{*}\right)$-compact.

(iv) The closed absolutely convex hull of every null sequence in $(E, \mathcal{T})$ is $\mathcal{T}$-compact.

Observe that a hyperplane $H$ of the dual space $X^{*}$ of a Banach space $X$ is norm-closed and norming if, and only if, there exists $x^{* *} \in X^{* *} \backslash X$ such that $H=\operatorname{ker}\left\{x^{* *}\right\}$ (see, e.g., [FHHMZ, Exercise $3.88])$.

Lemma 9 Let $x^{* *} \in \ell_{\infty} \backslash c_{0}$. Let $H:=\operatorname{ker}\left\{x^{* *}\right\} \subset \ell_{1}$. If $\left(H, w\left(H, c_{0}\right)\right)$ is locally complete, then $\left(c_{0}, \mu\left(c_{0}, H\right)\right)$ is complete.

Proof: Assume that $\left(H, w\left(H, c_{0}\right)\right)$ is locally complete. Let $f: H \rightarrow \mathbb{R}$ be a linear function whose restriction to any absolutely convex and $w\left(H, c_{0}\right)$-compact subset of $H$ is $w\left(H, c_{0}\right)$-continuous. Observe that $f: H \rightarrow \mathbb{R}$ is $\|\cdot\|_{1}$-continuous (hence an element in the dual of $\left(H,\|\cdot\|_{1}\right)$ ): Indeed, if $\left\{h_{n}^{*}\right\}_{n=1}^{\infty}$ is a $\|\cdot\|_{1}$-null sequence in $H$, the fact that $\left(H,\|\cdot\|_{1}\right)$ is a Banach space shows that the 
subset $\overline{\Gamma\left\{h_{n}^{*}: n \in \mathbb{N}\right\}}\|\cdot\|_{1}$ of $H$ is $\|\cdot\|_{1}$-compact, hence $w\left(H, c_{0}\right)$-compact (and absolutely convex), and so $f\left(h_{n}^{*}\right) \rightarrow 0$ as $n \rightarrow \infty$.

Let $\widetilde{f}$ be a Hahn-Banach extension of $f$ to $\ell_{1}$, so $\widetilde{f} \in \ell_{\infty}$. For $n \in \mathbb{N}$, let $e_{n}^{*}$ be the $n$-th vector of the canonical basis of $\ell_{1}$. By the Grothendieck's completeness criterion $(\mathrm{G})$ above, the proof will be finished as soon as we show that $\widetilde{f} \in c_{0}$.

To this end, let us take a strictly increasing sequence $\left\{j_{n}\right\}_{n=1}^{\infty}$ of natural numbers with the property that $\lim _{n}\left\langle x^{* *}, e_{j_{n}}^{*}\right\rangle$ exits. Let us divide our argument in two cases:

Case 1: Assume that $\lim _{n}\left\langle x^{* *}, e_{j_{n}}^{*}\right\rangle=0$. Take $x^{*} \in \ell_{1}$ such that $\left\langle x^{* *}, x^{*}\right\rangle=1$. Then, for each $n \in \mathbb{N}$ the vector $z_{n}^{*}:=e_{j_{n}}^{*}-\left\langle x^{* *}, e_{j_{n}}^{*}\right\rangle x^{*}$ is in $H$, and clearly $\left\{z_{n}^{*}\right\}_{n=1}^{\infty}$ is a $w\left(H, c_{0}\right)$-null sequence. The local completeness of $\left(H, w\left(H, c_{0}\right)\right)$ implies that

$$
{\overline{\Gamma\left\{z_{n}^{*}: n \in \mathbb{N}\right\}}}^{w\left(H, c_{0}\right)}
$$

is a $w\left(H, c_{0}\right)$-compact subset of $H$ (Theorem 8$)$. Therefore

$$
f\left(z_{n}^{*}\right) \rightarrow 0 \text { as } n \rightarrow \infty,
$$

which, in particular, implies that $\left\{\widetilde{f}\left(e_{j_{n}}^{*}\right)\right\}_{n=1}^{\infty}$ is null.

Case 2: Assume now that $\lim _{n}\left\langle x^{* *}, e_{j_{n}}^{*}\right\rangle \neq 0$. Without loss of generality we can also assume that there exists $\varepsilon>0$ such that $\left|\left\langle x^{* *}, e_{j_{n}}^{*}\right\rangle\right|>\varepsilon$ for every $n \in \mathbb{N}$. Fix any double sequence $\left\{\left(n_{k}, m_{k}\right)\right\}_{k=1}^{\infty}$ of natural numbers such that $n_{k} \rightarrow \infty$ and $m_{k} \rightarrow \infty$. It is clear that the sequence $\left\{y_{k}^{*}\right\}_{k=1}^{\infty}$, defined by

$$
y_{k}^{*}:=\left\langle x^{* *}, e_{j_{n_{k}}}^{*}\right\rangle^{-1} e_{j_{n_{k}}}^{*}-\left\langle x^{* *}, e_{j_{m_{k}}}^{*}\right\rangle^{-1} e_{j_{m_{k}}}^{*}, \text { for } k \in \mathbb{N},
$$

is in $H$ and converges to 0 in the $w\left(H, c_{0}\right)$-topology. Again, the local completeness of $\left(H, \mu\left(H, c_{0}\right)\right)$ implies that

$$
B:={\overline{\Gamma\left\{y_{k}^{*}: k \in \mathbb{N}\right\}}}^{w\left(H, c_{0}\right)}
$$

is $w\left(H, c_{0}\right)$-compact (Theorem 8 ), and this shows that

$$
f\left(y_{k}^{*}\right) \rightarrow 0 \text { as } k \rightarrow \infty .
$$

Due to the fact that the sequences $\left\{n_{k}\right\}_{k=1}^{\infty}$ and $\left\{m_{k}\right\}_{k=1}^{\infty}$ diverge to $+\infty$ and are, otherwise, arbitrary, we get from (3) that $\left\{\left\langle x^{* *}, e_{j_{n}}^{*}\right\rangle^{-1} \widetilde{f}\left(e_{j_{n}}^{*}\right)\right\}_{n=1}^{\infty}$ is a Cauchy sequence, hence convergent, say to $l \in \mathbb{R}$. Therefore, if we put $\widetilde{g}:=\widetilde{f}-l x^{* *}$, we get that the sequence $\left\{\widetilde{g}\left(e_{j_{n}}^{*}\right)\right\}_{n=1}^{\infty}$ is null.

We claim that the value $l \in \mathbb{R}$ does not depend on the choice of the sequence $\left\{j_{n}\right\}_{n=1}^{\infty}$ as far as this sequence is strictly increasing and $\lim _{n}\left\langle x^{* *}, e_{j_{n}}^{*}\right\rangle$ exists and is not 0 . Indeed, take another such a sequence $\left\{i_{n}\right\}_{n=1}^{\infty}$. The previous argument shows that $\left\{\left\langle x^{* *}, e_{i_{n}}^{*}\right\rangle^{-1} \widetilde{f}\left(e_{i_{n}}^{*}\right)\right\}_{n=1}^{\infty}$ converges, say to $l^{\prime} \in \mathbb{R}$. Let $\left\{r_{n}\right\}_{n=1}^{\infty}$ be the strictly increasing sequence obtained by intercalating the sequences $\left\{j_{n}\right\}_{n=1}^{\infty}$ and $\left\{i_{n}\right\}_{n=1}^{\infty}$. The previous argument applied now to the sequence $\left\{e_{r_{n}}^{*}\right\}_{n=1}^{\infty}$ gives that $\left\{\left\langle x^{* *}, e_{r_{n}}^{*}\right\rangle^{-1} \widetilde{f}\left(e_{r_{n}}^{*}\right)\right\}_{n=1}^{\infty}$ converges again (indeed, the only thing we needed for getting the conclusion there is that the sequence $\left\{\left\langle x^{* *}, e_{r_{n}}^{*}\right\rangle\right\}_{n=1}^{\infty}$ is uniformly away from 0 , see formula (2)), hence $l=l^{\prime}$ and the claim is proved.

To conclude our proof, let us show that $\widetilde{g}$, which is an extension of $f$ to $\ell_{1}$ (and so agrees with $\widetilde{f}$ on $H$ ), is in $c_{0}$. Indeed, since $\widetilde{g}$ belongs to $\ell_{\infty}$, we can take a strictly increasing sequence of natural numbers $\left\{j_{n}\right\}_{n=1}^{\infty}$ such that $\left\{\widetilde{g}\left(e_{j_{n}}^{*}\right)\right\}_{n=1}^{\infty}$ converges, say to $a \in \mathbb{R}$. Since $x^{* *}$ is also in $\ell_{\infty}$, we can assume, passing if necessary to a subsequence, that $\left\{\left\langle x^{* *}, e_{j_{n}}^{*}\right\rangle\right\}_{n=1}^{\infty}$ converges, say to $b \in \mathbb{R}$. If $b=0$ we know, by Case 1 , that $\left\{\widetilde{f}\left(e_{j_{n}}^{*}\right)\right\}_{n=1}^{\infty}$ is null and so

$$
a=\lim _{n} \widetilde{g}\left(e_{j_{n}}^{*}\right)=\lim _{n}\left(\widetilde{f}\left(e_{j_{n}}^{*}\right)-l\left\langle x^{* *}, e_{j_{n}}^{*}\right\rangle\right)=0 .
$$


If, on the contrary, $b \neq 0$, by Case 2 we know that $\left\{\widetilde{g}\left(e_{j_{n}}^{*}\right)\right\}_{n=1}^{\infty}$ is null, so $a=0$. Since $\left\{\widetilde{g}\left(e_{n}^{*}\right)\right\}_{n=1}^{\infty}$ is a bounded sequence with zero as its unique cluster point, it converges to zero. Thus $\widetilde{g}$ belongs to $c_{0}$. It is enough to finalize the proof to observe that $\widetilde{f}$ and $\widetilde{g}$ coincide on $H$.

Lemma 10 Let $X$ be a separable nonreflexive Banach space that does not contain an isomorphic copy of $\ell_{1}$. Let $x^{* *} \in X^{* *} \backslash X$, and put $H:=\operatorname{ker}\left\{x^{* *}\right\}$. If $(X, \mu(X, H))$ is locally complete then $(H, \mu(H, X))$ is complete.

Proof: Let us assume that $(X, \mu(X, H))$ is locally complete. Take a linear map $f: X \rightarrow \mathbb{R}$ such that $\left.f\right|_{K}$ is $w(X, H)$-continuous for every absolutely convex and $w(X, H)$-compact subset $K$ of $X$. We claim that $f$ belongs to $H$. Then Grothendieck's completeness criterion $(\mathrm{G})$ above will conclude the proof. In order to prove the claim, observe first that $f$ is norm-continuous, so $f$ belongs to $X^{*}$. Since $X$ is separable and does not contain an isomorphic copy of $\ell_{1}$, there exists a sequence $\left\{x_{n}\right\}_{n=1}^{\infty}$ in $X$ that converges to $x^{* *}$ in the $w\left(X^{* *}, X^{*}\right)$-topology (see, e.g., [Di, p. 215]). It is clear that the sequence is $w(X, H)$-null. Since $\mu(X, H)$ is locally complete, then the set $B:=\overline{\Gamma(A)}^{w(X, H)}$ is $w(X, H)$-compact, where $A:=\left\{x_{n}: n \in \mathbb{N}\right\}$ (Theorem 8), hence $f$ restricted to $B$ is $w(X, H)$ continuous. Therefore, $\left\langle x_{n}, f\right\rangle \rightarrow 0$. Since $\left\langle x_{n}, f\right\rangle \rightarrow\left\langle x^{* *}, f\right\rangle$, we obtain that $f \in H$, and this proves the claim.

Remark 11 Lemma 10 implies, in particular, that $\left(H, \mu\left(H, c_{0}\right)\right)$ is complete if $\left(c_{0}, \mu\left(c_{0}, H\right)\right)$ is locally complete, where $H$ is a proper norming $\|\cdot\|_{1}$-closed hyperplane of $\ell_{1}$.

\section{Two more examples: $c_{0}$ and the James space $J$}

The space $c_{0}$ has no predual, hence there is no natural way to apply the former results to conclude completeness in the topology $\mu\left(c_{0}, H\right)$ for $\|\cdot\|_{1}$-closed norming subspaces $H$ of the dual. We show here that all $\|\cdot\|_{1}$-closed norming hyperplanes $H$ in $\ell_{1}$ give complete $\mu\left(c_{0}, H\right)$ topologies. We also consider the particular case of Banach spaces that are 1-codimensional in their bidual spaces.

In order to prove Proposition 13 we need the following Lemma.

Lemma 12 Let $X$ be a Banach space, and let $N$ be a norm-closed norming subspace of $X^{*}$. Then, every $w(X, N)$-bounded subset $S$ of $X$ is norm-bounded.

Proof. Consider the Banach space $(N,\|\cdot\|)$, where $\|\cdot\|$ denotes the norm on $N$ induced by the dual norm $\|\cdot\|$ on $X$. The set $\left.S\right|_{N}:=\left\{\left.x\right|_{N}: x \in S\right\}$ is a pointwise bounded set of continuous linear mappings on the Banach space $(N,\|\cdot\|)$. By the Banach-Steinhaus theorem the set $\left.S\right|_{N}$ is norm-bounded, i.e., there exists $C>0$ such that

$$
\sup \left\{\left|\left\langle x, b^{*}\right\rangle\right|: b^{*} \in B_{N}\right\} \leq C, \text { for all } x \in S .
$$

Due to the fact that $N$ is norming, there exists $B>0$ such that

$$
B\|x\| \leq \sup \left\{\left|\left\langle x, b^{*}\right\rangle\right|: b^{*} \in B_{N}\right\} \text { for all } x \in X .
$$

In particular, $B\|x\| \leq C$ for all $x \in S$, hence $\|x\| \leq C / B$ for all $x \in S$.

Proposition 13 (i) Let $N$ be a $\|\cdot\|_{1}$-closed and norming subspace of $\ell_{1}$. Then $\left(c_{0}, w\left(c_{0}, N\right)\right)$ is locally complete.

(ii) If $H$ is a proper $\|\cdot\|_{1}$-closed norming hyperplane of $\ell_{1}$, then $\left(c_{0}, w\left(c_{0}, H\right)\right)$ is a locally complete not sequentially complete space.

Proof: (i) Let $B$ be a closed disc in $\left(c_{0}, w\left(c_{0}, N\right)\right)$. Due to Lemma $12, B$ is a (closed) disc in $\left(c_{0},\|\cdot\|_{\infty}\right)$. Since $\left(c_{0},\|\cdot\|_{\infty}\right)$ is a Banach space, $B$ is a Banach disc (see the statement (B) above). The statement (A) above gives then the conclusion. 
(ii) There exists $x^{* *} \in \ell_{\infty} \backslash c_{0}$ such that $H:=\operatorname{ker}\left\{x^{* *}\right\}$. Let $y^{* *} \in \ell_{\infty}$ such that $y^{* *} \notin c_{0} \oplus$ $\operatorname{span}\left(x^{* *}\right)$. Let $\left\{x_{n}\right\}_{n=1}^{\infty}$ be a sequence in $c_{0}$ that $w\left(l_{\infty}, l_{1}\right)$-converges to $y^{* *}$. In particular, this sequence is $w\left(c_{0}, H\right)$-Cauchy. Assume that $\left\{x_{n}\right\}_{n=1}^{\infty}$ is $w\left(c_{0}, H\right)$ - convergent, say to $x \in c_{0}$. Then $y^{* *}-x$ belongs to $H^{\perp}\left(=\operatorname{span}\left\{x^{* *}\right\}\right)$, a contradiction. This proves that $\left(c_{0}, w\left(c_{0}, H\right)\right)$ is not sequentially complete. The local completeness of $\left(c_{0}, w\left(c_{0}, H\right)\right)$ follows from (i).

Corollary 14 Let $H$ be a $\|\cdot\|_{1}$-closed norming hyperplane of $\ell_{1}$. Then $\left(c_{0}, \mu\left(c_{0}, H\right)\right)$ is complete.

Proof. The space $\left(c_{0}, \mu\left(c_{0}, H\right)\right.$ is locally complete due to (i) in Proposition 13 and the fact that local completeness depend only on the dual pair (see (B) above). It follows from Lemma 10 that the space $\left(H, \mu\left(H, c_{0}\right)\right)$ is complete, in particular locally complete (see $(\mathrm{C})$ above). We can apply now Lemma 9 to get the conclusion.

The last two results deal with Banach spaces that are $n$-codimensional in their bidual spaces (such spaces are called quasireflexive in [CY]). An example of this situation for $n=1$ is given by James' space $J$ ([James], see also [FHHMZ, Def. 4.43]). It is worth noting that if a Banach space $X$ is $n$-codimensional in its bidual, then every $w^{*}$-dense norm-closed subspace of $X^{*}$ is norming (see, e.g., [FHHMZ, Exercise 5.5]).

We shall need the following result.

(D) [CY, Theorem 3.3] Let $X$ be a Banach space. The following statements are equivalent: (i) $X$ is quasireflexive of order $n$. (ii) There is an equivalent norm for $X$ such that $X^{*}=P \oplus R$, where $P$ is a $w^{*}$-dense norm-closed subspace such that the closed unit ball of $X$ is $w(X, P)$-compact and $R$ is an $n$-dimensional subspace of $X^{*}$.

Some details of the proof of (D) are needed below, so we provide here a sketch: First, assume that $X$ is quasireflexive of order $n$, say $X^{* *}=\pi(X) \oplus F$, where $\pi: X \rightarrow X^{* *}$ is the canonical injection and $F$ has dimension $n$. Put $P:=F_{\perp}\left(\subset X^{*}\right)$. The mapping $\phi: X \rightarrow P^{*}$ given by $\phi(x)\left(p^{*}\right):=\left\langle\pi(x), p^{*}\right\rangle$ for all $p^{*} \in P$ is readily seen to be an isomorphism onto. Define on $X$ the equivalent norm $|\|\cdot \mid\|$ given by $|\|x \mid\|:=\|\phi(x)\|$ for all $x \in X$. It is easy to see that the isometry $\phi:\left(X,\left\||\|\mid\|) \rightarrow\left(P^{*},\|\cdot\|\right)\right.\right.$ is $w(X, P)-w\left(P^{*}, P\right)$-continuous. Since $\left(B_{P^{*}}, w\left(P^{*}, P\right)\right)$ is compact, so it is $\left(B_{X}, w(X, P)\right)$.

Conversely, assume that $X^{*}=P \oplus R$ as in (ii). Let $R:=\operatorname{span}\left\{x_{k}^{*}: k=1,2, \ldots, n\right\}$ for some $x_{k}^{*} \in X^{*}, k=1,2, \ldots, n$. For $k=1,2, \ldots, n$, let $x_{k}^{* *} \in X^{* *}$ such that $\left.x_{k}^{* *}\right|_{P}=0$ and $\left\langle x_{k}^{* *}, x_{j}^{*}\right\rangle=$ $\delta_{k, j}$ for all $j=1,2, \ldots, n$. Then $P=\bigcap_{k=1}^{n} \operatorname{ker} x_{k}^{* *}$. Given $x^{* *} \in B_{\left(X^{* *},\|\cdot|\|\mid\|)\right.}$ there exists a net $\left\{x_{i}: i \in I, \leq\right\}$ in $B_{(X,\|\cdot\| \cdot \|)}$ that $w^{*}$-converges to $x^{* *}$. Since $B_{(X,\|\cdot\| \|)}$ is $w(X, P)$-compact, there exists $x \in B_{(X,\|\cdot \mid\|)}$ such that $x_{i} \rightarrow x$ in the topology $w(X, P)$. Obviously, $x^{* *}-x \in P^{\perp}$. Note that $P^{\perp}=\operatorname{span}\left\{x_{k}^{* *}: k=1,2, \ldots, n\right\}$, hence $x^{* *} \in X \oplus \operatorname{span}\left\{x_{k}^{* *}: k=1,2, \ldots, n\right\}$. It follows that $X^{* *}=X \oplus \operatorname{span}\left\{x_{k}^{* *}: k=1,2, \ldots, n\right\}$.

Proposition 15 Let $n \in \mathbb{N}$ and let $X$ be a Banach space which is $n$-codimensional in its bidual. Then, for every norm-closed and $w^{*}$-dense $n$-codimensional subspace $P \subset X^{*}$, the space $(X, w(X, P))$ is sequentially complete, and $(P, \mu(P, X))$ is complete.

Proof: Let $F:=P^{\perp}$. Then $X^{* *}=X \oplus F$. Let $\||\cdot| \mid$ be the equivalent norm from the result (D). It follows that $B_{(X,\|\cdot \mid\|)}$ is $w(X, P)$-compact. In particular, $(X, w(X, P))$ is sequentially complete. Observe that $\mu(P, X)$ is thus the topology of the norm on $P$, and so it is certainly complete.

A locally convex space $(E, \mathcal{T})$ is said to be a Mackey space if $\mathcal{T}$ coincides with the Mackey topology $\mu\left(E, E^{*}\right)$, where $E^{*}$ denotes the topological dual of $(E, \mathcal{T})$. The second part of the following result should be compared with [BP, Theorems 5.2.1 and 5.2.4].

Corollary 16 Let $X$ be a Banach space which is 1-codimensional in its bidual. Then $(X, \mu(X, H))$ is complete for every proper norm-closed and $w^{*}$-dense hyperplane $H \subset X^{*}$. In particular, if $X$ is separable, then no proper norm-closed and norming hyperplane $H$ of $X^{*}$ is a Mackey space.

Proof: Denote by $E$ the locally convex space $(X, \mu(X, H))$ and let $x^{* *} \in X^{* *}$ be such that $H=$ $\operatorname{ker}\left\{x^{* *}\right\} \subset X^{*}$. Then $x^{* *} \in X^{* *} \backslash X$. Let $\widehat{E}$ denote the completion of $E$, and let $f \in \widehat{E}$. 
Observe that $\widehat{E} \subset H^{*}$. Indeed, if $\left\{h_{n}^{*}\right\}_{n=1}^{\infty}$ is a sequence in $H$ that norm-converges to 0 then $B:=\overline{\Gamma\left\{h_{n}^{*}: n \in \mathbb{N}\right\}}$ is a norm-compact subset of $H$, hence $w(H, X)$-compact, and so $f\left(h_{n}^{*}\right) \rightarrow 0$. Let $\tilde{f} \in X^{* *}$ be a norm-continuous and linear extension of $f$ to $X^{*}$, i.e., $\tilde{f} \in X^{* *}$. Then, there exist $x \in X$ and $\lambda \in \mathbb{R}$ such that $\widetilde{f}=x+\lambda x^{* *}$. This shows that $\tilde{f}\left(h^{*}\right)=f\left(h^{*}\right)=\left\langle x, h^{*}\right\rangle$ for all $h^{*} \in H$, hence $E$ is complete by Grothendieck's completeness criterion. For the second part of the statement, note that $H$ is $\mu\left(X^{*}, X\right)$-dense, so $\left(H,\left.\mu\left(X^{*}, X\right)\right|_{H}\right)$ is certainly non-complete. However, by Lemma 10 , the space $(H, \mu(H, X))$ is complete.

Question. We do not know of $w^{*}$-dense and $\|\cdot\|_{1}$-closed subspaces $Y$ of $\ell_{1}$ such that $\mu\left(c_{0}, Y\right)$ is not complete.

Acknowledgement: We thank the referee for his/her remarks and suggestions, that helped to improve the overall shape of the paper.

\section{References}

[BC] J. Bonet and B. Cascales, Non-complete Mackey topologies on Banach spaces, Bulletin of the Australian Mathematical Society, 81, 3 (2010), 409-413.

[FHHMZ] M. Fabian, P. Habala, P. Hájek, V. Montesinos, and V. Zizler, Banach Space Theory. The Basis for Linear and Nonlinear Analysis, CMS Books in Mathematics, Springer-Verlag, 2011.

[BP] P. Pérez-Carreras and J. Bonet, Barreled Locally Convex Spaces, North-Holland Mathematical Studies 131, North-Holland, 1987.

[CY] P. Civin and B. Yood, Quasi-reflexive spaces, Proceedings of the American Mathematical Society 8, No. 5 (1957), 906-911.

[Di] J. Diestel, Sequences and Series in Banach Spaces, GTM 92, Springer-Verlag, 1984.

[Fl] K. Floret, Weakly compact sets, LNM 801, Springer-Verlag, 1980.

[G] G. Godefroy, Boundaries of convex sets and interpolation sets, Math. Ann. 277 (1987), 173-184.

[James] R.C. James, On nonreflexive Banach space isometric with its second conjugate, Proc. Nat. Acad. Sci. USA 37 (1951), 174-177.

[Ko] G. Köthe, Topological Vector Spaces I, Springer-Verlag, 1969. 